\title{
Methods of analysis of hierarchies and enclosed linear convolutionns in problem of constraction of integrated parameters of the health-related quality of life
}

\author{
Marina P. Diakovich \\ Angarsk State Technical University \\ East-Siberian Institute of medical and ecological researches \\ Angarsk, Russia \\ marik914@rambler.ru \\ Ivan A. Finogenko \\ Irkutsk scientific center \\ Matrosov Institute of System Dynamics and Control Theory \\ Irkutsk, Russia
}

\begin{abstract}
In this article the method of constructing the integral indicator of health-related quality of life (HRQoL) as a multidimensional and heterogeneous system is offered. This method is based on the combined application of the hierarchy analysis method (HAM) and the enclosed linear convolution linear convolution. The structural model of HRQoL is constructed using a standardized Russian version of the MOS SF-36 health survey. The enclosed linear convolution allows transforming vector indicators of the vector scores of HRQoL scales to single average index which after rationing is considered as an integral indicator. The main problem of choosing the weighting coefficients for the enclosed linear convolution of the vector scores of HRQoL scales is solved using the HAM. This method allows researchers to convert the qualitative characteristics of the structural model of HRQoL into quantitative ones. The received results can be used for integral assessment of HRQoL of the individual as object of management in health care and social policy for decision making on its improvement.
\end{abstract}

Keywords- helth-related quality of life; structural model method of hierarchy analysis method; matrix of pair wise comparisons; enclosed linear convolution; integrated parameters

\section{INTRODUCTION}

In present article authors continue researches of health related quality of life (HRQoL) at the individual level [1]. The concept «quality of a life» attracts the attention of physicians and sociologists, economists and psychologists. It differs from the concept of "standard of living" which describes the level of consumption of goods and to meet the basic vital needs of people. The standard of living can be measured fairly objectively, based on normative documents and statistical data. At the same time quality of a life is determined by subjective perception of the physical, psychological, emotional and social functioning. The subjective perception can be described only qualitatively. According to definition of the World Health Organization (WHO) "health is physical, mental, and social well-being, not only absence of disease". Therefore, if to speak about HRQoL, it is necessary to consider these three its components. Certainly, all of them are interconnected and each of them has own characteristics and criteria.

Medico-social research, including structured interview on the basis of the MOS SF-36 health survey, is one of the most common ways of measuring HRQoL components at the individual level [2]. The questionnaire contains 36 items grouped in 8 scales. Parameters of each scale vary between 0 and 100 points, where lower scores means more disability, higher scores means less disability

The all scales form two generalized parameters: PHC - the general component of physical health and MHC - the general component of mental health.

The general component of physical health includes physical functioning, role limitations due to physical health problems, bodily pain, and general health perceptions.

The general component of mental health includes vital activity (vitality, energy or fatigue), social functioning role limitations due to emotional problems, general mental health, covering psychological distress and well-being

Thus, we have 8 private scales which parameters vary between 0 both 100 points, and 2 general scales which give integrated characteristic of HRQOL.

Our purpose is to construct integrated parameter of HRQOL, as scalar function from parameters of 8 private scales (the bottom level of model). Values of this function and weight factors after normalization will be concluded between 0 and 1 . It should be noted that 100 points for parameters of all private scales mean complete the absence of restrictions or violations of the health of a person. At the same time, the integrated parameter should accept value 1 . We reach the set goal by combining two well-known methods-the hierarchy 
analysis method (HAM) and the enclosed linear convolution method which will be submitted below.

\section{RESULTS AND DISCUSSION}

\section{A. Work accomplished}

For achievement of the purpose of our research it is necessary to solve multi-criteria analysis task of definition of the relative importance of criteria of researched system. The decision of this task by simple method of expert survey which possesses only the information of qualitative character, inevitably introduces elements of subjectivity, and hence, can lead to serious mistakes. One of modern ways of one of converting of the qualitative information about object in quantitative (in weight factors) is HAM which has arisen in the middle of the last century in works of American mathematics T. Saaty [3, 4]. HAM has well recommended itself in many areas of human activity - economy, power and even politics. But in medicine and sociology this method has not yet found a proper application. First step HAM is decomposition of researched object and construction of its hierarchical model. For model of HRQOL we shall stop on the three-level hierarchical model built on the basis of MOS SF-36 health survey. The first level of model contains a unique component and the purpose of hierarchy - HRQOL. The second level contains two components: PHC and MHC, each of which decomposes into 4 components of the private scales forming the third and last level of hierarchy (Fig.1)

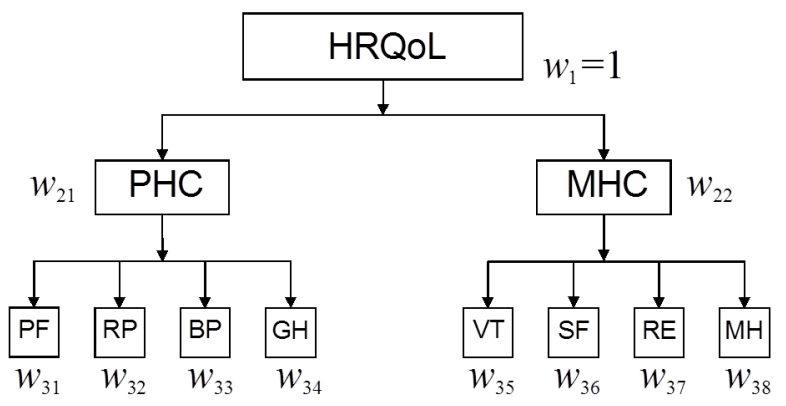

Fig.1 Hierarchical model of HRQOL

The criteria of scales are compared in pairs at each level of hierarchy and the matrix of pair comparisons of criteria is drawn up. This matrix has elements with the values of intensity of preferences of the criterion of one scale in relation to the criterion of another scale. For the model of HRQOL described above, we shall receive one square matrix of dimension 2 at the second level and two matrices with dimension 4- at the third level of model. At the first level we have a trivial matrix of dimension 1 which will not be further considered.

The number of criteria compared by experts should not exceed 9 (the psychological barrier of an expert). In our case this requirement is carried out. The matrix of pair comparisons possesses inverse symmetry property: $a_{i j}=1 / a_{j i}$ i.e. elements that are arranged symmetrically about the main diagonal of the matrix are mutually inverses, and units are elements of the diagonal. The vector $w=\left(w_{1}, w_{2}, \mathrm{~K}, w_{n}\right)$ of weight factors is the decision of the linear equation

$$
A w=\lambda_{\max } w,
$$

Where $\lambda_{\max }$ - the greatest eigenvalue of matrix $A$ and its deviation from dimension of matrix can be the measure of consistency of matrix. The validity and no contradictory of paired comparisons are determinate by the consistency of matrix. This measure is called the consistency relation (CO) and it is considered comprehensible if ones do not exceed 10 $\%$.

The enclosed linear convolution looks like

$$
J(w, x)=w_{21} \sum_{i=1}^{4} w_{3 i} x_{3 i}+w_{22} \sum_{i=5}^{8} w_{3 i} x_{3 i}
$$

Where ${ }^{x_{3 i}}$ - result of the person's answers to the questions of MOS SF-36 health survey in points from 0 up to 100 for scales of the third level of hierarchy, $w_{21}, w_{22}$ - weight factors of two scales of the second level $w_{3 i}, i=1, \mathrm{~K}, 8$, weight factors of scales of the third level, enclosed between 0 both 1 and received with use HAM .

The essence of the enclosed linear convolution lies in the fact that the components of the convolutions of the higher level of the hierarchy are the previously constructed convolutions of the lower levels, starting from the lowest level. It allows to increase dimension of any researched systems and to take into account influence of criteria of the bottom levels on higher level to the purpose of hierarchical model. form

Integrated parameter of HRQOL is presented in the

$$
I(w, x)=J(w, x) / 100
$$

Values of the integrated parameter lay between 0 and 1 . HRQOL of person will be better than him parameter $I(w, x)$ will be closer to 1 .

The value equal 1 , integrated parameter accepts only provided that parameters of all private scales of the bottom level of hierarchy HRQOL have accepted values of 100 points. Hence, equality $I(w, x)=1$ means the absence of restrictions or violations of the health of the person.

If values $I(w, x)$ are located between 0 and 1 the integrated parameter gives some average characteristic of a condition of the person. Thus parameters of private scales of the bottom level of hierarchy HRQOL can accept set of values, each of which corresponds to the same value of the integral parameter. This set of indicators is called the equivalence class.

\section{B. Work in progress}

Use of vector criteria of the estimation of a condition 
of researched multivariate object inevitably leads to occurrence of incomparable conditions. Scalarization of vector criteria [5] as linear convolution solves this problem, but thus we come to some the average characteristic of a condition of object. There are also other ways of scalarization of vector criteria of quality of the object, each of which has the advantages and lacks. The choice of suitable function represents an independent conceptual task.

Here we shall specify that constructed above linear convolution and the integrated parameter can serve also to serve as a feedback between the subject and object of control for improvement of a condition of object. In this case $I(w, x)$ it is considered as goal function. Its greatest value is known - it equally 1 . Therefore the problem of optimum way of achievement of this value arises only. Any function of vector argument grows fastly in a direction of the gradient. In this case it will be a vector of weight factors. Therefore for the fastest achievement by an integrated parameter of the greatest value it is necessary to change parameters of scales of the third level of hierarchy proportionally to their weight factors. It is necessary that all parameters of scales of the bottom level of model reach the values equal of 100 points. Thus, the factor of proportionality should be same for all parameters. If one or several parameters reach the greatest value equal 100 , other parameters should be changed only. The task is solved for the finite number of steps which are not exceeding 8 . It can form a basis for statement of others optimization tasks.

At last we shall note that decomposition and a choice of structural model HRQOL can be other [6]. Parameters and criteria of elements of HRQOL, together with a kind of an integrated parameter will be change accordingly.

\section{CONCLUSIONS}

The results obtained in the study, can be used for integrated estimation HRQOL at the individual level as object of control in health care and social policy and for decision making on HRQOL improvement. For this purpose it is necessary to construct an integrated parameter having executed the following actions:

1. To construct hierarchical model. Here we have stopped on the model based MOS SF-36 health survey. Matrixes of pair comparisons are defined.

2. To find weight factors from the solution of the equation (1) and CO. For this purpose the approached methods $[3,4]$ are used. If $\mathrm{CO}$ is unsatisfactory for any matrix this matrix is reconsidered.

3. To write down the enclosed linear convolution (2) and the integrated parameter (3).

Let's note that all these actions can be easily algorithmized and automated. But the compilation of comparison matrices cannot be fully formalized and demands the careful expert decision.

\section{REFERENCES}

[1] M. Diakovich, I. Finogenko, and A. Blokhin, "Information support of health-related quality of life of patients with occupational diseases", Proc. of Intern. Conf. "Information Technologies in Science, Management, Social Sphere and Medicine» (ITSMSSM 2016). Tomsk, 2016, pp. 153-156.

[2] J.E. Ware, C.D. Sherbour, "The MOS 36-item short form health survey: conceptual framework and item selection", Medical Care, 1992, Vol. 30, pp. 473-483.

[3] T. Saaty,"Decision making with dependencies and feedbacks. Analytical networks", Moscow: Book House "LIBROKOM", 1991, 357 p.

[4] T. Saaty, K. Kerns,"Analytical planning. Organization of systems", Moscow: Radio and communication, 2011, 224 p.

[5] I.G. Chernorutsky, "Method of optimization and decision-making", St. Petersburg: the Fallow deer, 2001, pp.41-44.

[6] V.S. Rukavishnikov, "Quality of life associated with health", Irkutsk: East-Siberian Scientific Center for Human Ecology, Siberian Branch of the Russian Academy of Medical Sciences, 2012, pp. 117-126. 